\title{
Visual position discrimination: A model relating temporal and spatial factors'
}

This paper presents a decision theory model of the perceptual processes by which an observer compares two visual stimuli presented at different points in time and at different locations in the visual field. The model specifies how information about the first stimulus is lost during the interstimulus delay and over the spatial translation required for the comparison. Emphasis is placed on the manner in which the effect of temporal separation combines with the effect of spatial separation in determining the observer's sensitivity. Two experiments are reported. The observer was required to discriminate a difference in vertical position between two laterally separated points of light presented successively in the dark. The progressive loss in sensitivity with increasing temporal and spatial separations is consistent with the predictions of the model.

Suppose an observer is presented with a stimulus at one point in his visual field and then, some time later, presented with a second stimulus at a different point in the field. Most readers would probably agree that his accuracy in judging the similarity of the two stimuli would be reduced by both the temporal delay between their occurrence and their spatial separation in his visual field. For example, consider the advantage one gains in comparing the color of two objects if they are viewed in immediate temporal succession and/or close together in space. Increasing either the temporal delay or the spatial separation between the two objects makes it more difficult to perceive whether the two colors are the same or different. This suggests that an observer's ability to judge the similarity of two objects is limited not only by their physical similarity but also by the manner in which they are observed.

In this paper, a decision theory model is developed to represent the effect of temporal and spatial factors on an observer's ability to compare two visual stimuli. The model is applied to data from a visual position discrimination task. The observer was required to discriminate a difference in vertical position between two laterally separated points of light presented successively in the dark. The variables of primary interest in this task were the length of the delay between successive presentations of the two stimuli, and the degree of lateral separation between them.

Decision theory models in psychophysics have largely addressed themselves to discrimination problems in which the two stimuli are temporally and spatially contiguous. Such discrimination tasks are generally referred to as detection problems. The most prominent of these models is the Theory of Signal Detection (see Green \& Swets, 1966, for a summary of detection models). This is a two-process model in that an observer's performance in a discrimination task is represented as the product of two subprocesses: an input process and a decision process. The input process relates the external stimulus event to hypothetical sensory states. The particular sensory state evoked is treated as a value of a random variable whose distribution depends on the physical properties of the actual stimulus value and the sensory capacities of the observer. A measure of the observer's "sensitivity" characterizes the input process. This measure is based on the relationship between the two distributions of sensory states evoked by the two stimulus events to be discriminated. The decision process relates the random variable representing the sensory states to the observer's overt response. An observer's tendency or "bias" to make a particular response is influenced by such experimenter controlled events as the presentation probabilities of the stimuli, and the costs or gains associated with the various stimulus-response contingencies. The bias is characterized by a single variable, the "decision criterion." The observer's overt response is based on the relation between the value of the random variable evoked by a particular stimulus event and his decision criterion. While an observer may modify his performance by altering his decision criterion, the measure of his sensitivity will not be affected. It is the separation of previously confounded "sensitivity" and "response bias" measures which is the major accomplishment of the decision theory approach to the analysis of psychophysical data.

A two-process model like the Theory of Signal Detection makes no provision for specifying changes in an observer's sensitivity produced by variations in the temporal delay between the two stimuli. Nor does it specify changes in sensitivity produced by variations in the spatial separation between the two stimuli.

Kinchla and Smyzer (1967), Ronken (1967), Tanner, Haller, and Atkinson (1967), and Tanner (1961) have developed models which represent the perceptual processes by which an observer compares stimulus events presented at different points in time. These models are concerned with the problem of memory in psychophysics, and have been denoted as recognition models (Kinchla, 1966; Kinchla \& Smyzer, 1967; and Tanner, Haller, \& Atkinson, 1967) in order to distinguish them from detection models. Of special interest here is Kinchla's diffusion model of perceptual memory (Kinchla \& Smyzer, 1967). This model is a three-process model in that recognition is represented as the product of three subprocesses: an input process, a memory process, and a decision process. As in the Theory of Signal Detection, the input process specifies the manner in which the physical properties of the stimulus event and the sensory capacity of the observer determine the hypothetical sensory state evoked by the stimulus event. The memory process considers the manner in which information about the initial stimulus is lost or degenerated during the interstimulus interval. The sensory state evoked by the first stimulus event is stored in memory where it is "diffused" through a random walk process during the interstimulus interval. The observer is represented as basing his response on the discrepancy between his memory of the sensory state evoked by the initial stimulus, and the sensory state evoked by the second stimulus. The decision process specifies how a given discrepancy interacts with the observer's decision criterion to determine his overt response. The observer reports a stimulus difference only if the discrepancy exceeds his decision criterion.

Keller and Kinchla (1968), Kinchla and Allan (1968), and Kinchla and Smyzer (1967) applied this model to data from a visual recognition task. The observer was required to discriminate a lateral difference in position between two small points of light flashed successively in the dark with a variable time interval between flashes. The progressive loss in sensitivity predicted by the model for increasing delays between successive presentations of the two lights, was shown to be consistent with the observed data.

Keller and Kinchla, Kinchla and Allan, and Kinchla and Smyzer suggested that the loss of position information represented by the theoretical random walk process may to a large extent reflect overt, involuntary eye movements during the interval between the two flashes. Support for this interpretation is found in studies concerned with direct measurement of eye movements in the dark. For example, Cornsweet (1956), and Matin (cited as personal communication by Kinchla \& Smyzer, 1967) have reported that the drift components of involuntary movements of the eye in the dark suggest a random walk process. If the loss of position information in the experiments described by Kinchla and his associates is primarily a result of eye movements during the interstimulus interval, then the model proposed by Kinchla would allow a measure of eye drift to be derived from purely psychophysical data.

As mentioned earlier, it can readily be demonstrated that an 
observer's efficiency in discriminating the color of two visual stimuli decreases as the spatial separation between them increases. However, no quantitative theory of this relationship is available. The present paper presents an elaboration of Kinchla's model to represent the manner in which information about one stimulus is lost during a spatial translation required to compare it with another stimulus. Furthermore, the model specifies the manner in which the effect of spatial separation combines with the effect of temporal separation in determining an observer's ability to discriminate two stimuli. The loss of information during the spatial translation is represented by the same mathematical process that is used to represent the loss of information over time in the Kinchla model. Therefore, since the loss due to time is attributed to imperfect perceptual memory in the Kinchla model, the loss due to space will be characterized as a perceptual memory effect in this model. On the basis of the distinction between detection and recognition made earlier, this model will be denoted as a recognition model.

One other aspect of the discrimination process will be considered empirically, although no theoretical treatment will be proposed. The majority of decision theory models in psychophysics treat the observer's performance as a series of stochastically independent trials. That is, it is usually assumed that the observer's response on any given trial is independent of the stimuli and responses which occurred on previous trials. However, several investigators (Atkinson, Carterette, \& Kinchla, 1962; Atkinson \& Kinchla, 1965; Kinchla, 1964, 1966; Parducci \& Sandusky, 1965; Tanner, Haller, \& Atkinson, 1967) have reported data from a number of experimental tasks which do not support this assumption. They have shown that the observer's response tendencies on a given trial are correlated with the stimulus and the response on the previous trial. Of particular interest here are the large and orderly sequential effects found in recognition tasks (Kinchla, 1966; Parducci \& Sandusky, 1965; Tanner et al, 1967). These far exceeded the sequential effects typically found in detection experiments. In fact, it has been pointed out that the most striking disparity between recognition and detection data is the sequential structure of the observer's performance (Kinchla, 1966). Since the perceptual memory process provides the primary distinction between detection and recognition models, the sequential structure of the data collected in the present experiments was examined in order to further explore the relationship between the memory and decision processes.

\section{A MATHEMATICAL MODEL OF THE PERCEPTUAL PROCESS}

In this section a model for the comparison of temporally separated stimuli (Kinchla \& Smyzer, 1967) will be elaborated to represent the comparison of spatially separated stimuli as well. Before introducing the theoretical issues, it will be helpful to specify the experimental situation and introduce some notation.

The discrimination experiments we shall consider consist of a series of discrete trials. On each trial the observer is shown two serially presented points of light, $L_{0}$ and $L_{1}$ respectively, which may or may not differ along a particular stimulus dimension. The observer's task is to report a stimulus difference when one exists. We shall denote the relevant stimulus variable by $Y$, and the values of $L_{0}$ and $L_{1}$, by $y_{0}$ and $y_{1}$ respectively. On some trials, $y_{1}$ equals $y_{0}$; on others $y_{1}$ differs from $y_{0}$ by an amount $y$. The following

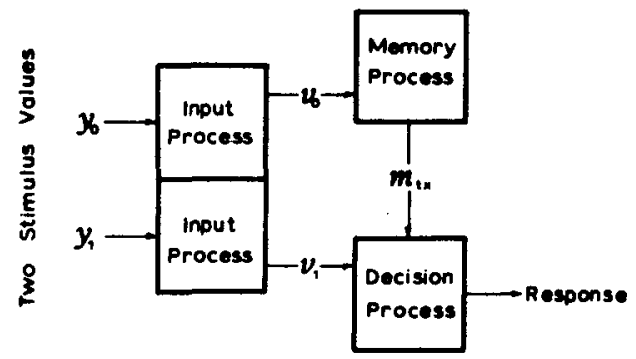

Fig. 1. Schematic of the model.

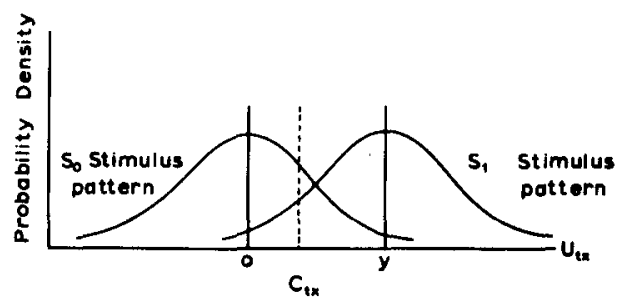

Fis. 2. Distribution of $U_{t x}$ conditional upon the difference between $y_{0}$ and $\mathbf{y}_{1}$.

notation will be used to describe the stimulus-response combina tions on Trial $n$ :

$S_{1, n}=$ the presentation of a stimulus difference on Trial $n$,

$S_{0, n}=$ the presentation of no stimulus difference on Trial $n$,

$A_{1, n}=$ the observer's response that a stimulus difference occurred on Trial $n$,

$A_{0, n}=$ the observer's response that no stimulus difference occurred on Trial $n$.

Thus, on each trial either $S_{1}$ or $S_{0}$ is presented, and the observer makes either an $A_{1}$ or $A_{0}$ response. Note that his performance can be summarized by two proportions: the proportion of $S_{1}$ trials on which he makes an $A_{1}$ response, for $i$ equal to 1 or 0 . These proportions are normally treated as estimates of corresponding conditional probabilities; respectively, $\mathbf{P}\left(\mathbf{A}_{1} \mid \mathbf{S}_{1}\right)$ and $P\left(A_{1} \mid S_{0}\right)$. In keeping with the decision theory literature, an $A_{1}$ response made to an $S_{1}$ stimulus pattern will be called a hit $(\mathrm{H})$, and an $A_{1}$ response made to $S_{0}$ will be called a false alarm (FA).

The purpose of the model is to account for changes in an observer's ability to discriminate a stimulus difference y produced by variations of two variables: the temporal interval between the offset of $L_{0}$ and the onset of $L_{1}$, denoted $t$; and the spatial separation between $L_{0}$ and $L_{1}$, denoted $x$. The basic structure of the model is shown schematically in Fig. 1. Each time some value of the stimulus variable $Y$ initiates the input process it evokes some value of the sensory variable $V$. The values of $V$ evoked by $y_{0}$ and $y_{1}$ are denoted respectively by $v_{0}$ and $v_{1}$. Since $y_{1}$ occurs later in time and/or spatially separated from $y_{0}$, the observer stores $v_{0}$ in "memory" until $v_{1}$ is available for comparison. He then makes a similarity decision regarding $y_{0}$ and $y_{1}$ on the basis of the size of the discrepancy between his memory of $v_{0}$, denoted $\mathrm{m}_{\mathrm{tx}}$, and $\mathrm{v}_{1}$. He reports a stimulus difference only if the observed discrepancy exceeds his decision criterion. Thus, three processes interact to determine the relationship between stimulus and response: input, memory, and decision. While these three processes can be defined in a rigorous axiomatic manner, a simple, verbal presentation should suffice here.

\section{The Input Process}

Repeated inputs of the same stimulus value do not necessarily evoke the same sensory value. The distribution of evoked sensory values is assumed to be Gaussian with an expected value equal to the actual stimulus value. Thus, if the same stimulus values $y_{0}$ and $y_{1}$ were presented on every discrimination trial, the sensory values $v_{0}$ and $v_{1}$ on any particular trial could be treated as values of two corresponding, independent, Gaussian, random variables, $V_{0}$ and $V_{1}$ respectively, where

$$
\begin{aligned}
& E\left(V_{0}\right)=y_{0}, \\
& E\left(V_{1}\right)=y_{1} .
\end{aligned}
$$

It is assumed that the variances of the two distributions, $\operatorname{Var}\left(V_{0}\right)$ and $\operatorname{Var}\left(V_{1}\right)$, are equal. Their sum will be referred to as the input variance. (It will be shown later that the equality of variance assumption is reasonable for the experiments to be considered.)

\section{The Memory Process}

Once the sensory value $v_{0}$ is stored in memory, it is "diffused" 
or modified through two simultaneous but independent random walk processes until it is read into the decision process. One random walk occurs during the interstimulus interval; the other during the spatial translation of the information provided by the initial stimulus. Note that $m_{t x}$, the memory of $v_{0}$, depends solely on the initial value in memory $\left(v_{0}\right)$ plus the cumulative effect of each random walk. These cumulative effects, denoted $d_{1}$ and $d_{x}$ respectively, are simply the sum of all the incremental steps minus the sum of all the decremental steps. The value of the memory can be written as

$$
m_{t x}=v_{0}+d_{t}+d_{x} \text {. }
$$

Since the decision process operates on the difference between $v_{1}$ and $m_{t x}$, it will be useful to denote this discrepancy by $u_{t x}$, where

$$
u_{t x}=v_{1}-m_{t x},
$$

or by Equation 3

$$
u_{t x}=v_{1}-v_{0}-d_{t}-d_{*} .
$$

If the same stimulus values $y_{0}$ and $y_{1}$ are presented on every discrimination trial, the values $d_{t}, d_{x}, m_{t x}$, and $u_{t x}$ on any particular trial can be treated as values of four corresponding, random variables, $D_{t}, D_{x}, M_{t x}$, and $U_{t x}$. Since the expected value of the sum of $n$ independent, random variables is the algebraic sum of their expected values, then from Equation 4,

$$
E\left(U_{t x}\right)=E\left(V_{1}\right)-E\left(V_{0}\right)-E\left(D_{t}\right)-E\left(D_{x}\right) \text {. }
$$

Furthermore, since the variance of the sum of $n$ independent, random variables equals the sum of their individual variances, then from Equation 4,

$$
\operatorname{Var}\left(U_{t x}\right)=\operatorname{Var}\left(V_{1}\right)+\operatorname{Var}\left(V_{0}\right)+\operatorname{Var}\left(D_{t}\right)+\operatorname{Var}\left(D_{x}\right)
$$

If the random walks are symmetrical, then as the number of steps in the random walks increases, the distributions of $D_{t}$ and $D_{x}$ (actually binomial) will approach Gaussian distributions with the following means and variances (Allan, 1968):

$$
\begin{aligned}
& E\left(D_{t}\right)=0, \\
& E\left(D_{x}\right)=0, \\
& \operatorname{Var}\left(D_{t}\right)=\phi_{t} t, \\
& \operatorname{Var}\left(D_{x}\right)=\phi_{x} x,
\end{aligned}
$$

where $\phi_{t}$ and $\phi_{x}$ are constants. The constant $\phi_{t}$, which we shall refer to as the temporal diffusion rate, is the rate at which the variance of $D_{t}$ increases as a linear function of the interstimulus interval (t). Similarly, $\phi_{x}$, the spatial diffusion rate, is the rate at which the variance of $D_{x}$ increases as a linear function of the spatial displacement between the two stimulus lights $(x)$.
Since $U_{t x}$ is defined as a linear combination of four independent, Gaussian, random variables (Equation 4), it too will have a Gaussian distribution. The expected value of $U_{t x}$ depends on the actual pair of stimulus values; that is, substituting in Equation 5 using Equations 1, 2, 7, and 8 yields,

$$
E\left(U_{t x}\right)=y_{1}-y_{0} \text {. }
$$

Furthermore, substituting in Equation 6 using Equations 9 and 10 yields,

$$
\operatorname{Var}\left(U_{t x}\right)=\operatorname{Var}\left(V_{1}\right)+\operatorname{Var}\left(V_{0}\right)+\phi_{t} t+\phi_{x} x .
$$

Thus, the variance of the $U_{t x}$ is the simple sum of the input variance plus the diffusion variance accrued in memory. It will be convenient to denote $\operatorname{Var}\left(U_{t x}\right)$ by the symbol $\sigma^{2}$ tx and the input variance by the symbol $K$. Thus,

$$
\sigma^{2} t x=\phi_{t} t+\phi_{x} x+K .
$$

\section{The Decision Process}

The observer's task is to decide whether the discrepancy $\left(u_{t x}\right)$ on a particular trial was produced by an actual stimuhus difference. For example, suppose $y_{1}$ equalled $y_{0}$ (an $S_{0}$ stimulus pattern) on a randomly determined 50 per cent of the trials, and equalled $y_{0}$ phus y (an $S_{1}$ stimulus pattern) on the remaining trials. The observer's decision problem is illustrated in Fig. 2, which presents two overlapping, Gaussian distributions of $U_{t x}$. The distribution with mean zero corresponds to $S_{0}$ trials, while the mean $y$ distribution corresponds to $S_{1}$ trials (Equation 11). The observer is assumed to establish some cutoff point or criterion value of $U_{t x}$, denoted $C_{t x}$, and to report a stimulus difference only if the observed discrepancy exceeds $C_{t x}$; specifically,

$$
\begin{aligned}
& P\left(A_{1, n} \mid u_{t x}>C_{t x}\right)=1, \\
& P\left(A_{1, n} \mid u_{t x}<C_{t x}\right)=0 .
\end{aligned}
$$

\section{Some Properties of the Model}

From Fig. 2 it can be seen that $P\left(A_{1} \mid S_{1}\right)$ is the area to the right of $C_{t x}$ under the $S_{1}$ distribution; similarly, $P\left(A_{1} \mid S_{0}\right)$ is the area to the right of $C_{t x}$ under the $S_{0}$ distribution. Note that although an

\begin{tabular}{|c|c|c|c|c|c|c|c|c|c|c|c|c|c|}
\hline \multirow[b]{2}{*}{$\underline{y}^{0}$} & \multirow[b]{2}{*}{$x^{0}$} & \multicolumn{2}{|c|}{ Observer 1} & \multicolumn{2}{|c|}{ Observer 2} & \multicolumn{2}{|c|}{ Observer 3} & \multicolumn{2}{|c|}{ Observer 4} & \multicolumn{2}{|c|}{ Observer 5} & \multicolumn{2}{|c|}{ Observer 6} \\
\hline & & $\hat{\mathbf{P}}\left(A_{1} \mid S_{1}\right)$ & $\hat{\mathrm{P}}\left(\mathrm{A}_{1} \mid \mathrm{S}_{0}\right)$ & $\hat{\mathrm{P}}\left(\mathrm{A}_{\mathbf{1}} \mid \mathrm{S}_{\mathbf{1}}\right)$ & $\hat{\mathbf{P}}\left(\mathbf{A}_{1} \mid \mathrm{S}_{0}\right)$ & $\hat{\mathbf{P}}\left(\mathbf{A}_{1} \mid \mathbf{S}_{1}\right)$ & $\hat{\mathbf{P}}\left(\mathbf{A}_{1} \mid \mathrm{S}_{0}\right)$ & $\hat{\mathbf{P}}\left(\mathbf{A}_{\mathbf{1}} \mid \mathrm{S}_{\mathbf{1}}\right)$ & $\hat{\mathbf{P}}\left(\mathbf{A}_{\mathbf{1}} \mid \mathbf{S}_{0}\right)$ & $\hat{\mathbf{P}}\left(\mathrm{A}_{1} \mid \mathrm{S}_{1}\right)$ & $\hat{\mathbf{P}}\left(\mathrm{A}_{1} \mid \mathrm{S}_{0}\right)$ & $\hat{\mathbf{P}}\left(\mathbf{A}_{1} \mid \mathbf{S}_{\mathbf{1}}\right)$ & $\hat{\mathbf{P}}\left(\mathbf{A}_{1} \mid \mathbf{S}_{0}\right)$ \\
\hline .26 & 2 & .72 & .01 & .83 & .36 & .86 & .08 & .88 & .24 & .90 & .16 & .99 & .16 \\
\hline .26 & 4 & .67 & .01 & .82 & .43 & .89 & .19 & .82 & .32 & .85 & .23 & .98 & .26 \\
\hline .26 & 6 & .56 & .02 & .70 & .37 & .84 & .23 & .76 & .38 & .77 & .27 & .91 & .33 \\
\hline .26 & 8 & .52 & .05 & .66 & .37 & .82 & .41 & .78 & .50 & .75 & .30 & .91 & .39 \\
\hline .26 & 10 & .45 & .10 & .66 & .48 & .88 & .49 & .72 & .43 & .71 & .30 & .85 & .41 \\
\hline 13 & 2 & .20 & .00 & .67 & .44 & .54 & .12 & .58 & .29 & .70 & .28 & .93 & .21 \\
\hline .13 & 4 & .22 & .03 & .58 & .41 & .51 & .19 & .63 & .40 & .73 & .28 & .88 & .35 \\
\hline .13 & 6 & .29 & .05 & .55 & .38 & .61 & .26 & .65 & .41 & .66 & .33 & .83 & .38 \\
\hline .13 & 8 & .34 & .13 & .60 & .44 & .64 & .44 & .59 & .45 & .63 & .38 & .77 & .46 \\
\hline .13 & 10 & .32 & .11 & .55 & .44 & .74 & .47 & .57 & .49 & .55 & .36 & .74 & .48 \\
\hline
\end{tabular}
observer may produce many combinations of $P\left(A_{1} \mid S_{1}\right)$ and $P\left(A_{1} \mid S_{0}\right)$ by shifting his criterion, he can never change $P\left(A_{1} \mid S_{1}\right)$ without simultaneously changing $P\left(A_{1} \mid S_{0}\right)$. The possible pairs of hit and false alarm rates available to the observer through variation in his decision criterion are his operating characteristic (OC). The $O C$ can be specified by a single number, the sensitivity measure $\delta_{t x}$, defined as follows:

$$
\delta_{t x}=\frac{E_{1}\left(U_{t x}\right)-E_{0}\left(U_{t x}\right)}{\left[\operatorname{Var}\left(U_{t x}\right)\right]^{1 / 2}},
$$

where $E_{0}\left(U_{t x}\right)$ is the expected value of $U_{t x}$ on $S_{0}$ trials, and $E_{1}\left(U_{t x}\right)$ is the expected value on $S_{1}$ trials. Thus $\delta_{t x}$ is simply the distance between the means of the two distributions of $U_{t x}$

Estimated Values of $P\left(A_{1} \mid S_{1}\right)$ and $P\left(A_{1} \mid S_{0}\right)$ for Each Observer Under Each Experimental Condition in Experiment 1 
Table 2

Estimated Values of $\mathbf{P}(\mathrm{C})$ for Each Observer Under Each Experimental Condition in Experiment 1

\begin{tabular}{rrrrrrrr}
\hline$y^{\circ}$ & $x^{\circ}$ & Obs. 1 & Obs. 2 & Obs. 3 & Obs. 4 & Obs. 5 & Obs. 6 \\
\hline .26 & 2 & .86 & .73 & .89 & .82 & .87 & .92 \\
.26 & 4 & .83 & .70 & .85 & .74 & .81 & .86 \\
.26 & 6 & .77 & .66 & .80 & .69 & .75 & .79 \\
.26 & 8 & .74 & .65 & .70 & .64 & .72 & .76 \\
.26 & 10 & .68 & .59 & .69 & .64 & .70 & .72 \\
& & & & & & & \\
.13 & 2 & .60 & .61 & .70 & .64 & .71 & .86 \\
.13 & 4 & .60 & .58 & .66 & .62 & .72 & .77 \\
.13 & 6 & .62 & .58 & .68 & .62 & .66 & .72 \\
.13 & 8 & .60 & .58 & .60 & .57 & .62 & .66 \\
.13 & 10 & .60 & .56 & .63 & .54 & .59 & .63 \\
\hline
\end{tabular}

expressed in standard deviation units. Substituting in Equation 13 from Equations 11 and 12 yields,

$$
\delta_{t x}=\frac{y}{\left(\phi_{t} t+\phi_{x} x+K\right)^{2 / 2}} .
$$

Note that in detection,

$$
\delta_{\mathrm{tx}}=\frac{\mathrm{y}}{\mathrm{K}^{1 / 2}} .
$$

Thus, when the two stimuli are contiguous in time and in space, the present model is equivalent to the Theory of Signal detection, and $\delta_{t x}$ and $\mathrm{d}^{\prime}$ (the sensitivity measure in that theory) are equivalent. In recognition, the variance introduced by an imperfect perceptual memory process $\left(\phi_{t} t+\phi_{x} x\right)$ is added to the input variance and reduces $\delta_{\mathrm{tx}}$.

\section{AN EMPIRICAL TEST OF THE MODEL}

We shall now consider two experiments which provide a test of the model. In order to increase the comparability of results, data for the two experiments were collected on alternate days with the same observers participating in both studies. During the experiments, the observer sat in complete darkness and tried to discriminate a difference in vertical position between two successively presented points of light. In terms of the model, the stimulus variable $Y$ corresponds to the vertical position of each light. The first experiment was designed to provide information about the manner in which an observer's sensitivity $\left(\delta_{t x}\right)$ is influenced by the size of the vertical displacement to be discriminated $(y)$, and by the extent of the spatial separation between the two stimulus lights $(x)$. Experiment 2 investigated the manner in which the effect of spatial separation $(x)$ combines with the effect of temporal separation ( $t$ ) in determining an observers' sensitivity.

Since the two experiments were similar in several respects, we will first consider the common features.

\section{Apparatus}

The stimuli were small points of light (Dialco No. $39,28 \mathrm{~V}, .04$ A, operated at $15 \mathrm{~V} \mathrm{dc}$ ) subtending $.033 \mathrm{deg}$ of visual angle at a brightness of $4 \mathrm{~mA}$. They were presented at approximately eye level, $4.1 \mathrm{~m}$ in front of an observer seated in a completely dark room. The observer sat in a normal chair with no special constraints on his head movements, and viewed the stimuli binocularly. The distance between the midpoints of two stimulus lights was specified in degrees of visual angle subtended by the lights. The timing of the stimulus presentations was electronically controlled to at least an accuracy of $1 \mathrm{msec}$, and the stimulus sequence was preprogrammed and fully automatic.

\section{Observers}

Six paid observers were used. Each observer had an uncorrected visual acuity of $20 / 20$ or better in both eyes according to the conventional Snellen visual acuity test. The observers were informed of the physical structure of the stimulus display, the random method for generating stimulus sequences, and the relative frequency of occurrence of the various stimulus patterns.

\section{Method}

Each discrimination trial began with a 1 sec auditory warning signal followed immediately by a $1 \mathrm{sec}$ presentation of a point of light, $\mathrm{L}_{0}$. After a $\mathrm{t} \sec$ delay, a second point of light, $\mathrm{L}_{\mathrm{i}}$, displaced $x$ deg to the right, was presented for $1 \sec . L_{1}$ was either at the same vertical height as $L_{0}$ (an $S_{0}$ stimulus pattern), or displaced $y$ deg down on the vertical axis (an $S_{1}$ stimulus pattern). Finally, the observer was given $2 \mathrm{sec}$ to indicate (by pressing an appropriate pushbutton on the arm of his chair) one of two decisions: $L_{1}$ occurred in the same vertical position as $L_{0}$ (an $A_{0}$ response); or $L_{1}$ was below $L_{0}$ (an $A_{1}$ response). The experimental variables were $t$ (the temporal interval between the two lights), $x$ (the lateral separation between the two lights), and $y$ (the size of the vertical displacement to be discriminated).

$\mathrm{L}_{0}$ occurred in the same position in space on every trial: a point at approximately eye level directly in front of the seated observer. It defined the origin of the two-dimensional, stimulus space. It is implicit in the present form of the model that the observer has a perfect conception of the vertical and horizontal dimensions. In an attempt to approximate this condition, an additional light was presented simultaneously with $L_{0}$. This light was 2 deg to the left of $L_{0}$ and at the same vertical height as $L_{0}$. Naturally, in the future it will be necessary to evaluate the importance of this additional cue.

\section{Experiment 1}

The sequence of stimulus presentations was separately determined for each block of 50 trials. In each such sequence an $S_{1}$ stimulus pattern was presented on a randomly determined 25 trials, while on the remaining 25 trials an $S_{0}$ pattern was presented. The interstimulus interval, $t$, was $0 \mathrm{sec}$ (the lights occurred in immediate temporal succession) throughout the experiment. During each test session $x(2,4,6,8$, or $10 \mathrm{deg})$ and $y$ (.26 or .13 deg) were varied between blocks of 50 trials. The sequence of the 10 experimental conditions was randomly determined within each session, and there was a $1 \mathrm{~min}$ rest in the dark between blocks of trials. The experiment consisted of 12 such test sessions. In this way, 12 blocks of 50 trials each were collected for each of the 10 conditions, for a total of 600 trials per experimental condition.

In addition, three preliminary days of testing were conducted to provide stable data for analysis. Also, in order to control warm-up effects, and to allow sufficient time for dark adaptation (about 10 min), two practice blocks of 50 trials each were conducted at the beginning of each session. The practice conditions were randomly determined with the limitation that every condition was used an equal number of times before any of the 10 conditions were repeated. The data from the three preliminary days and from the practice blocks were not included in the final data analysis.

\section{Experiment 2}

The size of the vertical displacement to be discriminated, $y$, was $.26 \mathrm{deg}$ throughout the experiment. During each test session $t(0$, $.333, .667$, or $1 \mathrm{sec})$ and $x(6$ or $10 \mathrm{deg})$ were varied between blocks of 50 trials. The experiment consisted of 12 such sessions. Thus, 12 blocks of 50 trials each were collected for each of the eight conditions for a total of 600 trials per experimental condition. In all other respects, Experiment 2 was identical to Experiment 1.

\section{RESULTS}

In this section we will present the data and statistically evaluate the effect of the independent variables in each experiment. A deeper analysis of the results is provided by the model and is presented in the subsequent section.

\section{Experiment 1}

Each observer's performance can be summarized by the average hit and false alarm proportions obtained for each of the 10 
experinental conditions. These proportions were treated as estimates of corresponding conditioning probabilities, denoted as $\hat{P}\left(A_{1} \mid S_{1}\right)$ and $\hat{P}\left(A_{1} \mid S_{0}\right)$, and are presented in Table 1 . An index of sensitivity frequently used in psychophysical experiments is the probability of a correct response, $\mathrm{P}(\mathrm{C})$. Estimates of $\mathrm{P}(\mathrm{C})$, denoted $\mathbf{P}(C)$, for each experimental condition, can readily be obtained from each observer's summary data in the following manner:

$$
\hat{\mathrm{P}}(C)=\hat{\mathrm{P}}\left(\mathrm{A}_{1} \mid \mathrm{S}_{1}\right) \gamma+\left[1-\hat{\mathrm{P}}\left(\mathrm{A}_{1} \mid \mathrm{S}_{0}\right)\right](1-\gamma),
$$

where $\gamma$ is the probability of an $S_{1}$ stimulus pattern. These estimates are presented in Table 2 for each observer. Note that, in general, $\hat{P}(C)$ tends to decrease with increases in the lateral separation between the two lights $(x)$, and is considerably greater for the larger vertical discrepancy $(y)$.

The effect of each independent variable on each observer's performance was evaluated by Chi-square homogeneity tests (Anderson \& Goodman, 1957, p. 97). Under the null hypothesis the expected number of $A_{j}$ responses made to $S_{i}$, for $i$ and $j$ equal to 1 or 0 , is the same for all values of the independent variable. Assuming the null hypothesis to be true, the best estimate of the expected frequency of an $A_{i}$ response to $S_{i}$ is the average frequency over all values of the variable. Given these expected frequencies, a Chi-square statistic may be calculated in the usual manner. Both the lateral separation between the two lights $(x)$ and the size of the vertical discrepancy to be discriminated $(y)$ had a statistically significant effect $(p<.001)$ on each observer's performance.

\section{Experiment 2}

For each observer, $\hat{\mathrm{P}}\left(\mathrm{A}_{1} \mid \mathrm{S}_{1}\right)$ and $\hat{\mathrm{P}}\left(\mathrm{A}_{1} \mid \mathrm{S}_{0}\right)$ for each of the eight experimental conditions are presented in Table 3 , and $\hat{P}(C)$ in Table 4. Note that $\hat{\mathbf{P}}(\mathrm{C})$ tends to decrease as the temporal interval between the two lights $(t)$ is increased, and is generally greater for the smaller lateral separation $(x)$.

As in Experiment 1, the effect of each independent variable on each observer's performance was evaluated by Chi-square homogeneity tests. With one exception, both the temporal delay $(t)$ and the lateral separation $(x)$ had a statistically significant effect $(p<.01)$ on each observer's performance. Manipulation of the lateral displacement between the two lights did not produce a significant influence $(p>.05)$ on the performance of Observer 2 . However the overall Chi-square for $x$, computed by summing the values for each observer, was significant $(p<.001)$.

\section{THEORETICAL ANALYSIS AND DISCUSSION}

Effects of the Independent Variables on Sensitivity $\left(\delta_{t}\right)$

For each observer an estimate of $\delta_{t x}$, denoted $\delta_{t x}$, can be obtained for each experimental condition from a table of normal deviates. Specifically,

$$
\hat{\delta}_{t x}=\hat{P}_{z}\left(A_{1} \mid S_{0}\right) \cdot \hat{P}_{z}\left(A_{1} \mid S_{1}\right)
$$

where $\hat{P}_{z}\left(A_{1} \mid S_{0}\right)$ is that value of a normal deviate which is exceeded with a probability $\hat{\mathbf{P}}\left(A_{1} \mid S_{0}\right)$, and $\hat{\mathbf{P}}_{z}\left(A_{1} \mid S_{1}\right)$ is a similar transformation of $\hat{P}\left(A_{1} \mid S_{1}\right)$. These estimates of $\delta_{t x}$ are presented
Table 4

Estimated Values of $P(C)$ for Each Observer Under Each Experimental Condition in Experiment 2

\begin{tabular}{rrrrrrrr}
\hline$x^{\circ}$ & $\mathrm{t} \mathrm{sec}$ & Obs. 1 & Obs. 2 & Obs. 3 & Obs. 4 & Obs. 5 & Obs. 6 \\
\hline 6 & .000 & .78 & .64 & .78 & .69 & .81 & .80 \\
6 & .333 & .74 & .61 & .71 & .61 & .75 & .79 \\
6 & .667 & .70 & .61 & .62 & .60 & .70 & .77 \\
6 & 1.000 & .66 & .57 & .62 & .59 & .63 & .68 \\
10 & .000 & .74 & .63 & .65 & .62 & .68 & .73 \\
10 & .333 & .66 & .62 & .64 & .61 & .64 & .69 \\
10 & .667 & .68 & .56 & .64 & .56 & .64 & .70 \\
10 & 1.000 & .64 & .57 & .64 & .55 & .63 & .66 \\
\hline
\end{tabular}

as data points in Fig. 3, and numerically in Tables 5 and 6 . It is clear that in general, the independent variables have a systematic effect on $\hat{\delta}_{\text {tx }}$ for each observer. In Experiment 2, the relationship between the two independent variables, $x$ and $t$, would have been more clearly demonstrated if the difference between the two values of $\mathrm{x}$ was greater. Note that $\hat{\delta}_{\mathrm{tx}}$ appears to be a more sensitive measure than $\hat{\mathbf{P}}(\mathrm{C})$ in that variations in the independent variables that do not produce an apparent change in $\hat{\mathbf{P}}(\mathrm{C})$, clearly affect $\hat{\delta}_{t x}$. For example, examine the performance of Observer 1 in Experiment 1 , y equal to $.13 \mathrm{deg}$. As $\mathrm{x}$ is increased from $2 \mathrm{deg}$ to $10 \mathrm{deg}, \hat{\delta}_{\mathrm{tx}}$ decreases from 1.48 to .76 (Table 5), while $\hat{\mathbf{P}}(\mathrm{C})$ varies only slightly around .60 (Table 2 ).

In order to evaluate the extent to which the estimates of $\delta_{t x}$ presented in Tables 5 and 6 are consistent with the values of $\delta_{t x}$ predicted by the model (Equation 14), estimates of the diffusion rates, $\phi_{t}$ and $\phi_{x}$, and of the input variance, $K$, have to be determined for each observer. If an observer's sensitivity does not vary between the two experiments, then these three parameters can be estimated from his performance in both experiments. Note that two of the experimental conditions in Experiment 1 were duplicated in Experiment 2 ( $x$ equal to $6 \mathrm{deg}$, y equal to $.26 \mathrm{deg}, \mathrm{t}$ equal to $0 \mathrm{sec}$; and $x$ equal to $10 \mathrm{deg}$, $y$ equal to $.26 \mathrm{deg}$, $t$ equal to 0 sec). A statistical test proposed by Gourevitch and Galanter (1967) was used to determine whether an observer's sensitivity under these two conditions in Experiment 1 differs significantly from his sensitivity under the corresponding conditions in Experiment 2. Specifically, the null hypothesis tested was that an estimate of $\delta_{t x}$ obtained in Experiment 1 does not differ significantly from the estimate of $\delta_{t x}$ obtained for the same experimental condition in Experiment 2. For each observer, two such tests were conducted. In only one case (Observer 5 ; $x$ equal to 6 $\mathrm{deg}$, y equal to $.26 \mathrm{deg}, \mathrm{t}$ equal to $0 \mathrm{sec}$ ) could the null hypothesis be rejected $(\mathrm{p}<.05)$. On the basis of these results, it can be argued that, in general, the variability in sensitivity between experiments is negligible.

Thus $\phi_{t}, \phi_{x}$, and K were estimated from an observer's performance in both experiments. These estimates, denoted respectively as $\hat{\phi}_{t}, \hat{\phi}_{x}$, and $\hat{\mathbf{K}}$, were chosen to minimize the sum of squared discrepancies between the 18 estimates of $\delta_{t x}$ obtained from the two experiments for a particular observer, and the

Table 3

\begin{tabular}{|c|c|c|c|c|c|c|c|c|c|c|c|c|c|}
\hline \multirow[b]{2}{*}{$x^{0}$} & \multirow[b]{2}{*}{$t \mathrm{sec}$} & \multicolumn{2}{|c|}{ Observer 1} & \multicolumn{2}{|c|}{ Observer 2} & \multicolumn{2}{|c|}{ Observer 3} & \multicolumn{2}{|c|}{ Observer 4} & \multicolumn{2}{|c|}{ Observer 5} & \multicolumn{2}{|c|}{ Observer 6} \\
\hline & & $\hat{\mathrm{P}}\left(\mathrm{A}_{1} \mid \mathrm{S}_{1}\right)$ & $\hat{\mathbf{P}}\left(\mathbf{A}_{1} \mid \mathbf{S}_{0}\right)$ & $\hat{\mathrm{P}}\left(\mathrm{A}_{1} \mid \mathrm{S}_{1}\right)$ & $\hat{\mathbf{P}}\left(\mathbf{A}_{1} \mid \mathrm{S}_{0}\right)$ & $\hat{\mathbf{P}}\left(\mathrm{A}_{1} \mid \mathrm{S}_{1}\right)$ & $\hat{\mathrm{P}}\left(\mathrm{A}_{1} \mid \mathrm{S}_{0}\right)$ & $\hat{\mathbf{P}}\left(\mathrm{A}_{1} \mid \mathrm{S}_{1}\right)$ & $\hat{\mathbf{P}}\left(\mathbf{A}_{1} \mid \mathrm{S}_{0}\right)$ & $\hat{\mathrm{P}}\left(\mathrm{A}_{1} \mid \mathrm{S}_{1}\right)$ & $\hat{\mathrm{P}}\left(\mathrm{A}_{1} \mid \mathrm{S}_{0}\right)$ & $\hat{\mathrm{P}}\left(\mathrm{A}_{1} \mid \mathrm{S}_{1}\right)$ & $\hat{\mathrm{P}}\left(\mathrm{A}_{1} \mid \mathrm{S}_{0}\right)$ \\
\hline 6 & .000 & .60 & .04 & .67 & .40 & .85 & .29 & .76 & .37 & .84 & .22 & .93 & .34 \\
\hline 6 & .333 & .54 & .06 & .64 & .42 & .69 & .26 & .74 & .52 & .77 & .28 & .91 & .33 \\
\hline 6 & .667 & .47 & .06 & .63 & .42 & .56 & .31 & .65 & .45 & .66 & .27 & .85 & .31 \\
\hline 6 & 1.000 & .41 & .09 & .56 & .41 & .52 & .28 & .70 & .52 & .60 & .34 & .74 & .37 \\
\hline 10 & .000 & .63 & .16 & .69 & .43 & .87 & .56 & .75 & .50 & .69 & .33 & .89 & .43 \\
\hline 10 & .333 & .42 & .10 & .66 & .41 & .83 & .55 & .77 & .55 & .69 & .40 & .82 & .44 \\
\hline 10 & .667 & .49 & .12 & .63 & .50 & .74 & .47 & .75 & .62 & .67 & .39 & .78 & .38 \\
\hline 10 & 1.000 & .39 & .11 & .57 & .42 & .62 & .34 & .64 & .54 & .62 & .36 & .70 & .37 \\
\hline
\end{tabular}

Estimated Values of $P\left(A_{1} \mid S_{1}\right)$ and $P\left(A_{1} \mid S_{0}\right)$ for Each Observer Under Each Experimental Condition in Experiment 2 

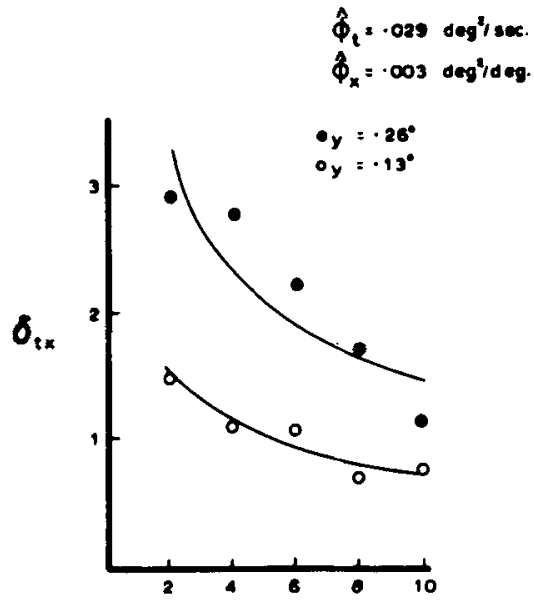

$x$ degrees

Observer 2

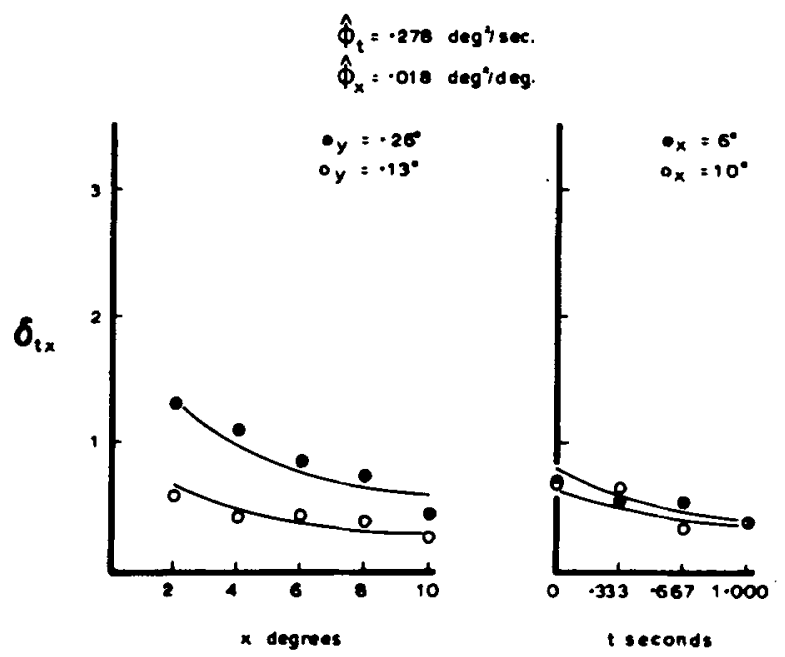

Observer 3

$\hat{\phi}_{t}=.120$ deg/enc.
$\hat{\phi}_{x}=.005$ degtoeg.
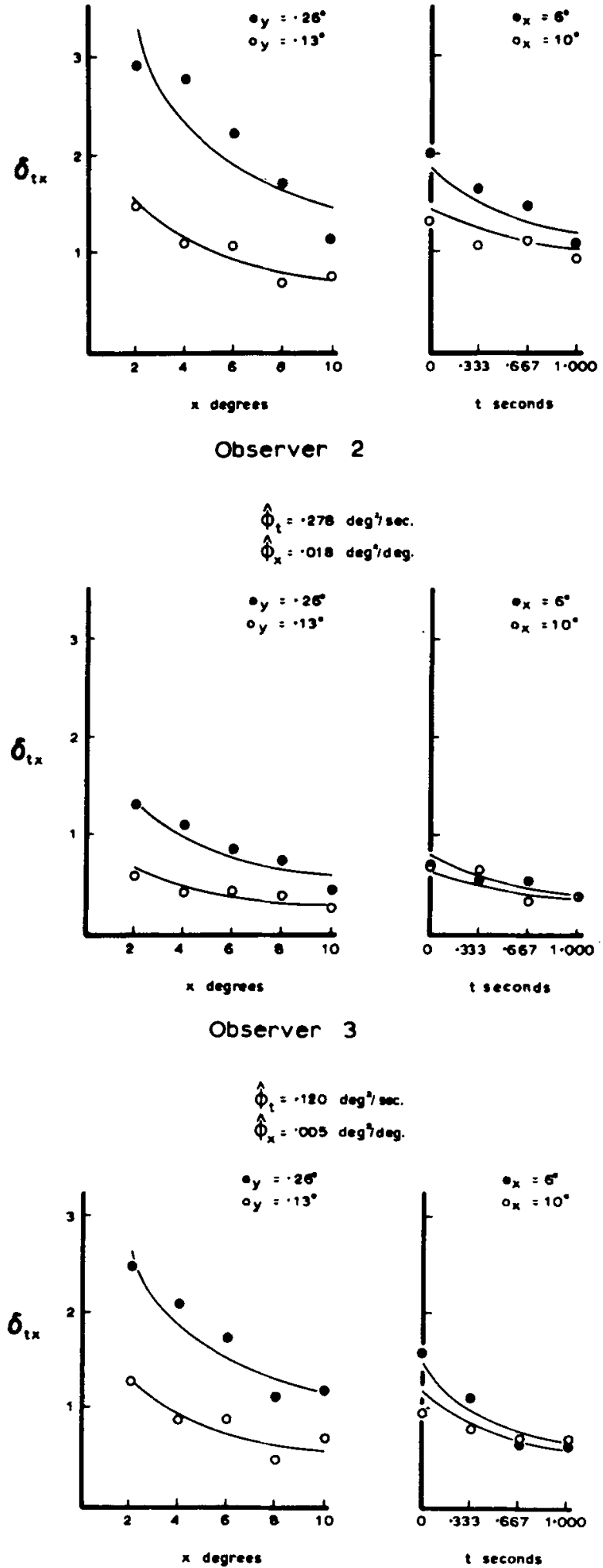

$\hat{\phi}_{\mathrm{t}}=-398 \operatorname{deg}^{2} / \mathrm{sec}$.

$\hat{\phi}_{x}=.011 \mathrm{deg} / \mathrm{deg}$.
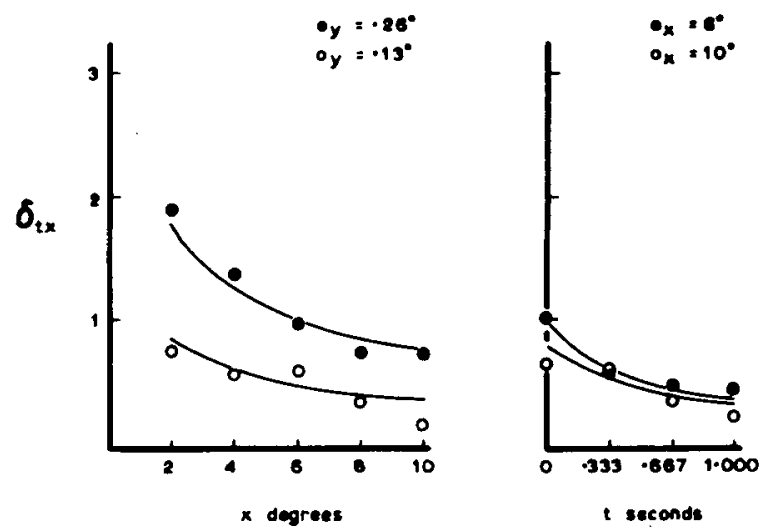

Observer 5

$\hat{\phi}_{t}=.074 \operatorname{deg}^{2} / \mathrm{sec}$.

$\hat{\phi}_{x}=.006 \mathrm{deg}^{2} / \mathrm{deg}$.
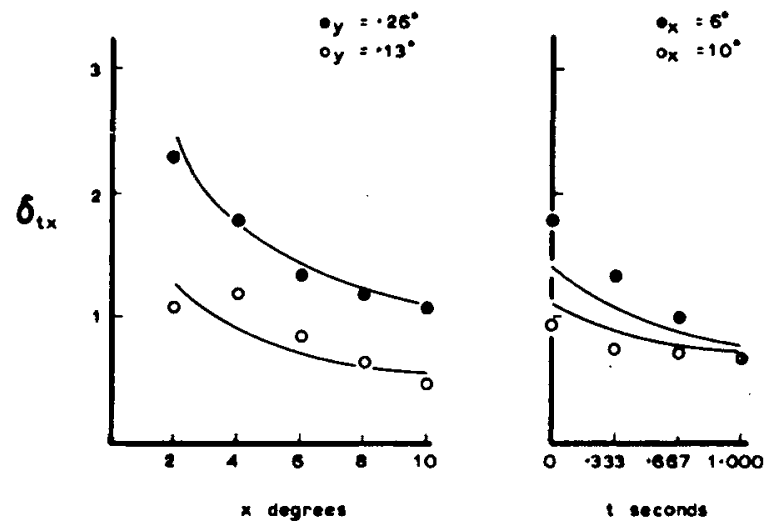

Observer 6

$\hat{\phi}_{\mathrm{t}}=.036 \mathrm{deg}^{2} / \mathrm{sec}$.

$\phi_{x}=.003$ degl/deg.
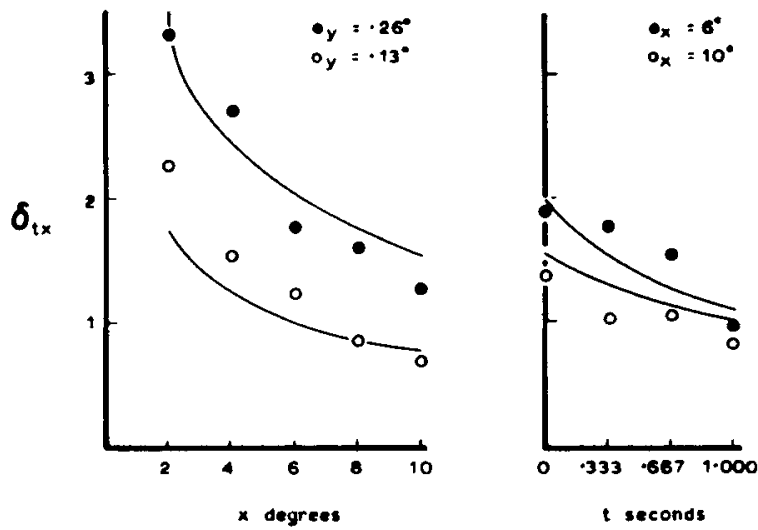

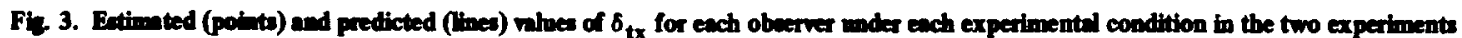
(2pencueter form of the model). 
Table 5

Estimated and Predicted ( 2 parameters) Values of $\delta_{1 x}$ for Each Observer Under Each Experimental Condition in Experiment 1

\begin{tabular}{|c|c|c|c|c|c|c|c|c|c|c|c|c|c|}
\hline \multirow[b]{2}{*}{$\mathrm{y}^{\circ}$} & \multirow[b]{2}{*}{$\mathbf{x}^{0}$} & \multicolumn{2}{|c|}{ Observer 1} & \multicolumn{2}{|c|}{ Observer 2} & \multicolumn{2}{|c|}{ Observer 3} & \multicolumn{2}{|c|}{ Observer 4} & \multicolumn{2}{|c|}{ Observer 5} & \multicolumn{2}{|c|}{ Observer 6} \\
\hline & & $\hat{\delta}_{t x}$ & Pred $\delta_{t x}$ & $\hat{\delta}_{\mathrm{tx}}$ & Pred $\delta_{t x}$ & $\overline{\hat{\delta}_{\mathrm{tx}}}$ & Pred $\delta_{t x}$ & $\hat{\delta}_{\mathrm{tx}}$ & Pred $\delta_{t x}$ & $\hat{\delta}_{t x}$ & Pred $\delta_{t x}$ & $\overline{\hat{\delta}_{t x}}$ & Pred $\delta_{t x}$ \\
\hline .26 & 2 & 2.90 & 3.25 & 1.31 & 1.37 & 2.48 & 2.65 & 1.88 & 1.77 & 2.27 & 2.46 & 3.31 & 3.47 \\
\hline .26 & 4 & 2.76 & 2.30 & 1.10 & .97 & 2.11 & 1.88 & 1.38 & 1.25 & 1.78 & 1.74 & 2.69 & 2.46 \\
\hline .26 & 6 & 2.20 & 1.88 & .86 & .79 & 1.73 & 1.53 & 1.01 & 1.02 & 1.35 & 1.42 & 1.78 & 2.00 \\
\hline .26 & 8 & 1.69 & 1.62 & .74 & .68 & 1.14 & 1.33 & .77 & .88 & 1.20 & 1.23 & 1.62 & 1.74 \\
\hline .26 & 10 & 1.15 & 1.45 & .46 & .61 & 1.20 & 1.19 & .76 & .79 & 1.08 & 1.10 & 1.27 & 1.55 \\
\hline .13 & 2 & 1.48 & 1.62 & .59 & .68 & 1.28 & 1.33 & .76 & .88 & 1.10 & 1.23 & 2.28 & 1.74 \\
\hline .13 & 4 & 1.11 & 1.15 & .43 & .48 & .90 & .94 & .58 & .62 & 1.19 & .87 & 1.56 & 1.23 \\
\hline .13 & 6 & 1.08 & .94 & .44 & .39 & .92 & .77 & .62 & .51 & .85 & .71 & 1.26 & 1.00 \\
\hline .13 & 8 & .72 & .81 & .40 & .34 & .51 & .66 & .36 & .44 & .64 & .61 & .84 & .87 \\
\hline .13 & 10 & .76 & .73 & .28 & .30 & .72 & .59 & .20 & .40 & .49 & .55 & .69 & .78 \\
\hline
\end{tabular}

corresponding values predicted by Equation 14 with $\hat{\phi}_{t}, \hat{\phi}_{x}$, and $\hat{k}$ substituted for $\phi_{t}, \phi_{x}$, and $K$. The input variance, $\hat{K}$, is negligible for all observers. In fact for Observers 4 and 6 the input variance is actually zero. This suggests an appropriate simplification of the model: let $K$ equal zero by assumption, and fit the data with two parameters, $\phi_{t}$ and $\phi_{x}$. The estimates of $\phi_{t}$ and $\phi_{x}$ presented in Fig. 3 were chosen to minimize the sum of squared discrepancies between the estimated $\delta_{t x}$ values and those predicted by Equation 14 with $\phi_{t}$ and $\hat{\phi}_{x}$ substituted for $\phi_{t}$ and $\phi_{x}$ and $K$ equal to zero. These predicted $\delta_{t x}$ values, denoted Pred. $\delta_{t x}$, are presented graphically in Fig. 3, and numerically in Tables 5 and 6 . It seems clear from Fig. 3 that the 2-parameter form of the model provides a reasonable interpretation of each observer's performance.

One way to test how well the model accounts for changes in sensitivity under the various experimental conditions is in terms of the proportion of the variance in $\hat{\delta}_{t x}$ accounted for by the predicted $\delta_{t x}$ values. The variance of the $18 \delta_{t x}$ values around their mean can be regarded as an estimate of the total variance in the dependent variable. An estimate of the unpredicted (or residual) variance in $\hat{\delta}_{\mathrm{tx}}$ is simply the variance of the $18 \hat{\delta}_{\mathrm{tx}}$ values about their predicted values minus the variance that can be attributed to sampling. Given an estimate of the observer's theoretical hit and false alarm probabilities, denoted $\hat{P}\left(A_{1} \mid S_{1}\right)$ and $P\left(A_{1} \mid S_{0}\right)$, an estimate of the sampling variance can be obtained using the method discussed by Gourevitch and Galanter (1967). That pair of hit and false alarm probabilities on the operating characteristic predicted by Equation 14 which was most similar to the observer's performance, was regarded as an estimate of his theoretical hit and false alarm probabilities. The proportion of the total variance accounted for by the model was calculated for each observer, and indicated that the model accounts on the average for.96 of the total variance in $\hat{\delta}_{t x}$ : the actual values obtained for Observers 1 through 6 are respectively $.96,1.00, .98,1.00, .94$, and .91 . Thus, the model accounts for virtually all of the variance in $\hat{\delta}_{t x}$. It is apparent that the two diffusion parameters allow a good prediction of $\hat{\delta}_{t x}$ under the 16 different experimental conditions.

In the development of the model it was assumed that $\operatorname{Var}\left(V_{0}\right)$ and $\operatorname{Var}\left(V_{1}\right)$, the variances of the two sensory distributions, were equal. Since the predictions of the two-parameter form of the model are consistent with the observed data, the equality of variance assumption appears to be reasonable, at least for the vertical position discrimination task considered.

\section{Estimates of Decision Criterion ( $\mathrm{C}_{\mathbf{t} \mathrm{x}}$ )}

Another theoretical question of interest is the relationship between variations in the values of the independent variables and an observer's decision criterion, $C_{t x}$. Note that $P\left(A_{1} \mid S_{0}\right)$ would by definition equal the area to the right of $\mathrm{C}_{\mathrm{tx}}$ under the distribution of $U_{t x}$ for an $S_{0}$ stimulus pattern (see Fig. 2). $P_{z}\left(A_{1} \mid S_{0}\right)$, that yalue of a normal deviate which is exceeded with a probability $P\left(A_{1} \mid S_{0}\right)$, can be obtained from a table of normal deviates. An estimate of the criterion, denoted $\hat{C}_{t x}$, in degrees visual angle is simply $P_{z}\left(A_{1} \mid S_{0}\right)$ multiplied by the standard error of $U_{t x}$. That is,

$$
\hat{\mathrm{C}}_{\mathrm{tx}}=\hat{\mathrm{P}}_{2}\left(\mathrm{~A}_{1} \mid \mathrm{S}_{0}\right) \sigma_{\mathrm{tx}}
$$

These estimates may be thought of as the minimum discrepancy in degrees visual angle that must occur between the memory of the first stimulus and the position of the second stimulus in order for the observer to respond "different."

Estimates of each observer's criterion for each experimental condition are presented in Figs. 4 and 5 . Since an estimate of the sampling variance is not available, we can not determine whether the observed variance in $\hat{C}_{t x}$ is greater than the variance that could be attributed to sampling. However, it is clear that, if part of the observed variance is attributable to variations in the independent variables, the relationship between the independent variables and $\hat{\mathrm{C}}_{\mathrm{tx}}$ is idiosyncratic. For example, the decision criterion held by Observer 3 is apparently dependent upon the value of $x$, but independent of the value of $y$. Observer 5 , however, seems to maintain a fairly stable criterion with variations in $x$, but holds a consistently smaller criterion for the smaller value of $y$. Also note that for Observer 3 the decision criterion is positively correlated with $t$, while for Observer 4 the correlation is negative. Thus the decision theory approach to the analysis of the data allows the

Table 6 Estimated and Predicted (2 parameters) Values of $\delta_{t x}$ for Each Observer Under Each Experimental Condition in Experiment 2

\begin{tabular}{|c|c|c|c|c|c|c|c|c|c|c|c|c|c|}
\hline \multirow[b]{2}{*}{$x^{0}$} & \multirow[b]{2}{*}{$t \sec$} & \multicolumn{2}{|c|}{ Observer 1} & \multicolumn{2}{|c|}{ Observer 2} & \multicolumn{2}{|c|}{ Observer 3} & \multicolumn{2}{|c|}{ Observer 4} & \multicolumn{2}{|c|}{ Observer 5} & \multicolumn{2}{|c|}{ Observer 6} \\
\hline & & $\hat{\delta}_{t x}$ & Pred $\delta_{\mathrm{tx}}$ & $\hat{\delta}_{t x}$ & $\operatorname{Pred} \delta_{\mathrm{tx}}$ & $\hat{\delta}_{t x}$ & Pred $\delta_{\text {tx }}$ & $\hat{\delta}_{\mathbf{t x}}$ & $\operatorname{Pred} \delta_{t x}$ & $\hat{\delta}_{t x}$ & Pred $\delta_{t x}$ & $\hat{\delta}_{\mathrm{tx}}$ & Pred $\delta_{\mathrm{tx}}$ \\
\hline 6 & .000 & 2.00 & 1.88 & .70 & .79 & 1.60 & 1.53 & 1.04 & 1.02 & 1.76 & 1.42 & 1.88 & 2.00 \\
\hline 6 & .333 & 1.65 & 1.53 & .56 & .58 & 1.14 & 99 & .59 & .61 & 1.32 & 1.08 & 1.78 & 1.53 \\
\hline 6 & .667 & 1.48 & 1.32 & .53 & .48 & .66 & .79 & .52 & .47 & 1.02 & .90 & 1.54 & 1.29 \\
\hline 6 & 1.000 & 1.11 & 1.18 & .38 & .42 & .63 & .67 & .48 & .40 & .66 & .79 & .97 & 1.13 \\
\hline 10 & .000 & 1.32 & 1.45 & .68 & .61 & .98 & 1.19 & .68 & .79 & .94 & 1.10 & 1.41 & 1.55 \\
\hline 10 & .333 & 1.08 & 1.27 & .64 & .50 & .82 & .88 & .61 & .54 & .76 & .92 & 1.06 & 1.30 \\
\hline 10 & .667 & 1.15 & 1.15 & .33 & .43 & .72 & .73 & .37 & .44 & .72 & .80 & 1.08 & 1.14 \\
\hline 10 & 1.000 & .95 & 1.05 & .38 & .38 & .72 & .63 & .26 & .38 & .66 & .72 & .86 & 1.03 \\
\hline
\end{tabular}



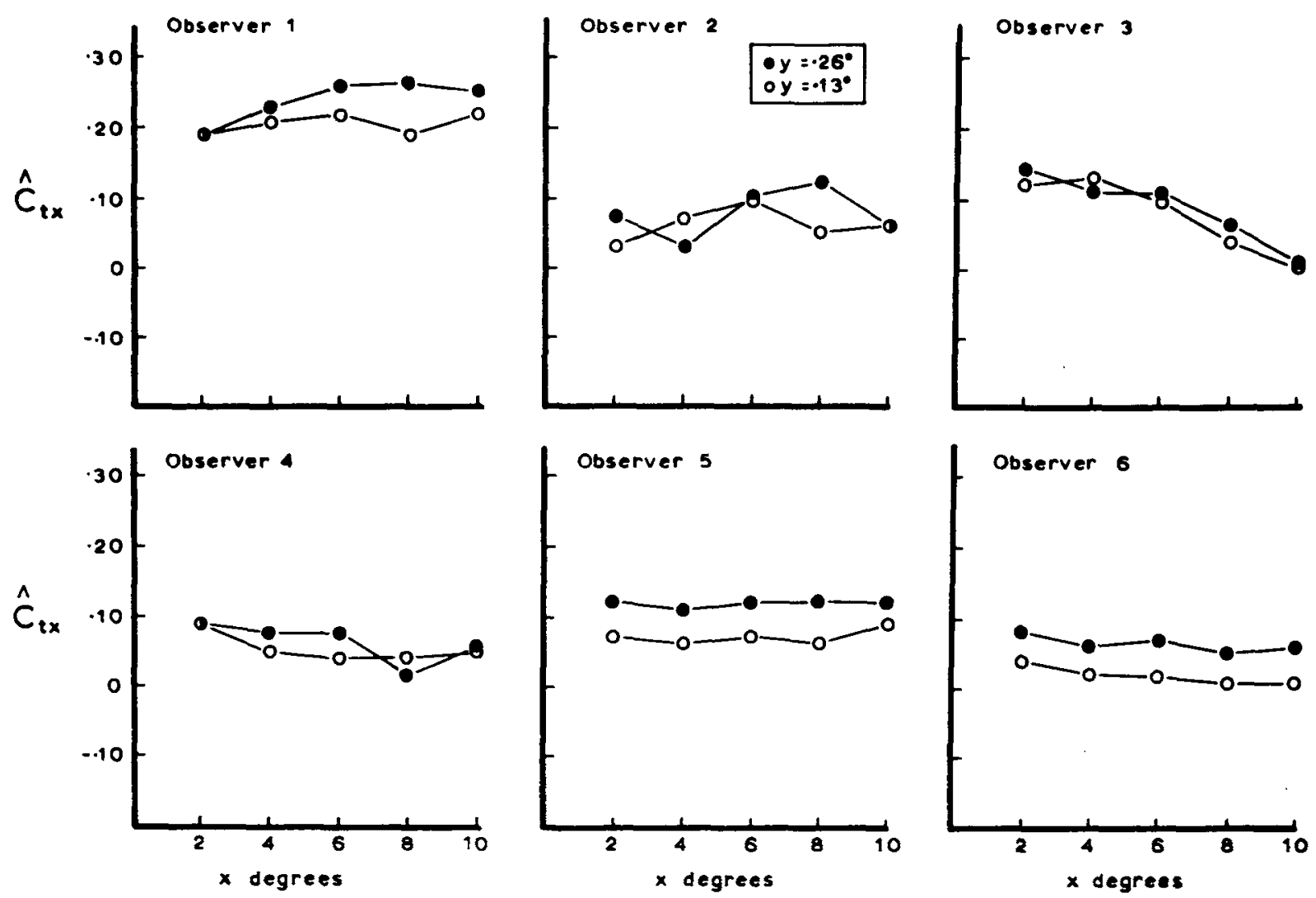

Fig. 4. Estimated values of $C_{\mathrm{tx}}$ for each observer under each experimental condition in Experiment 1.
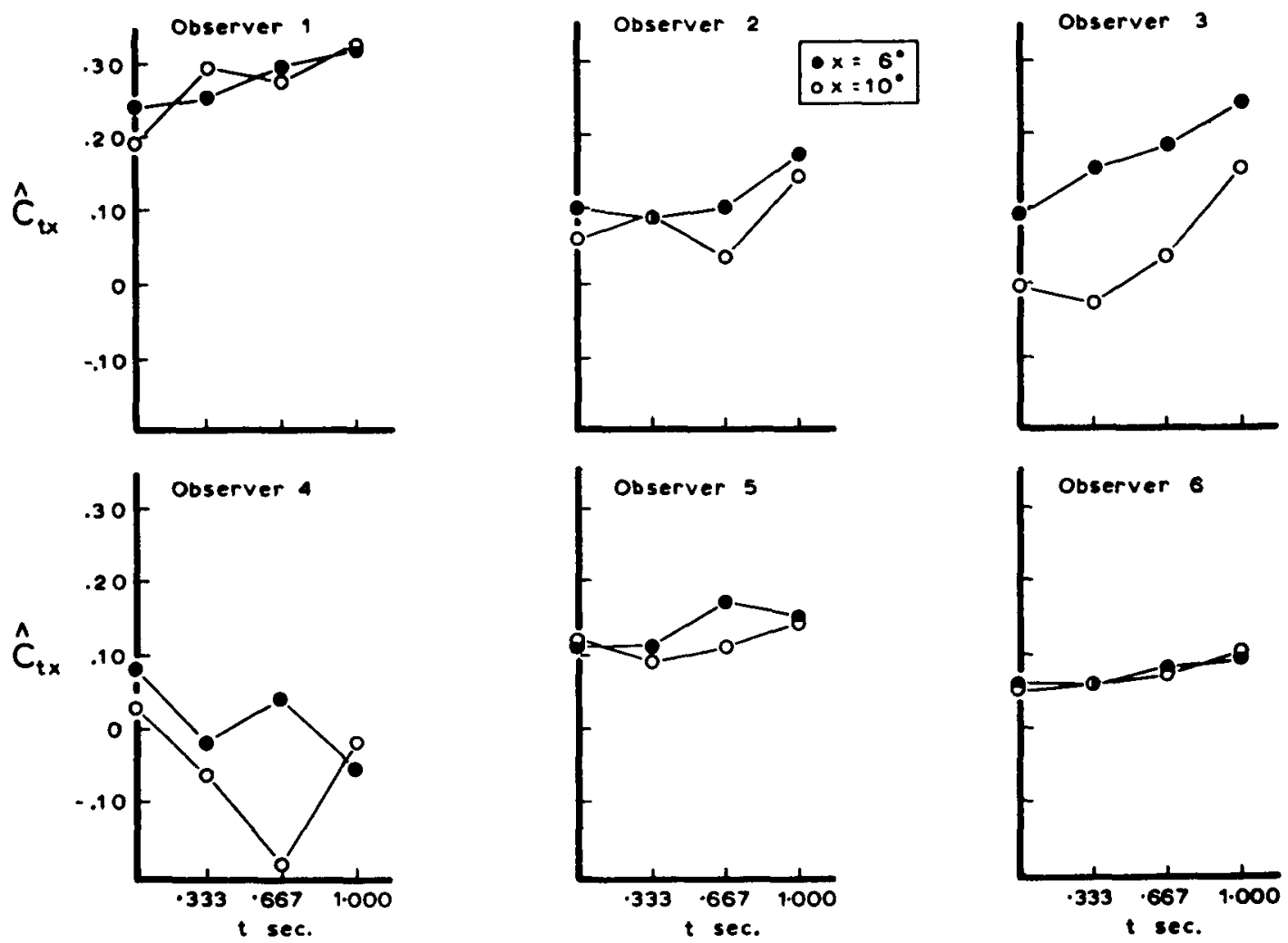

Fig. 5. Estimated values of $C_{t x}$ for each observer under each experimental condition in Experiment 2. 


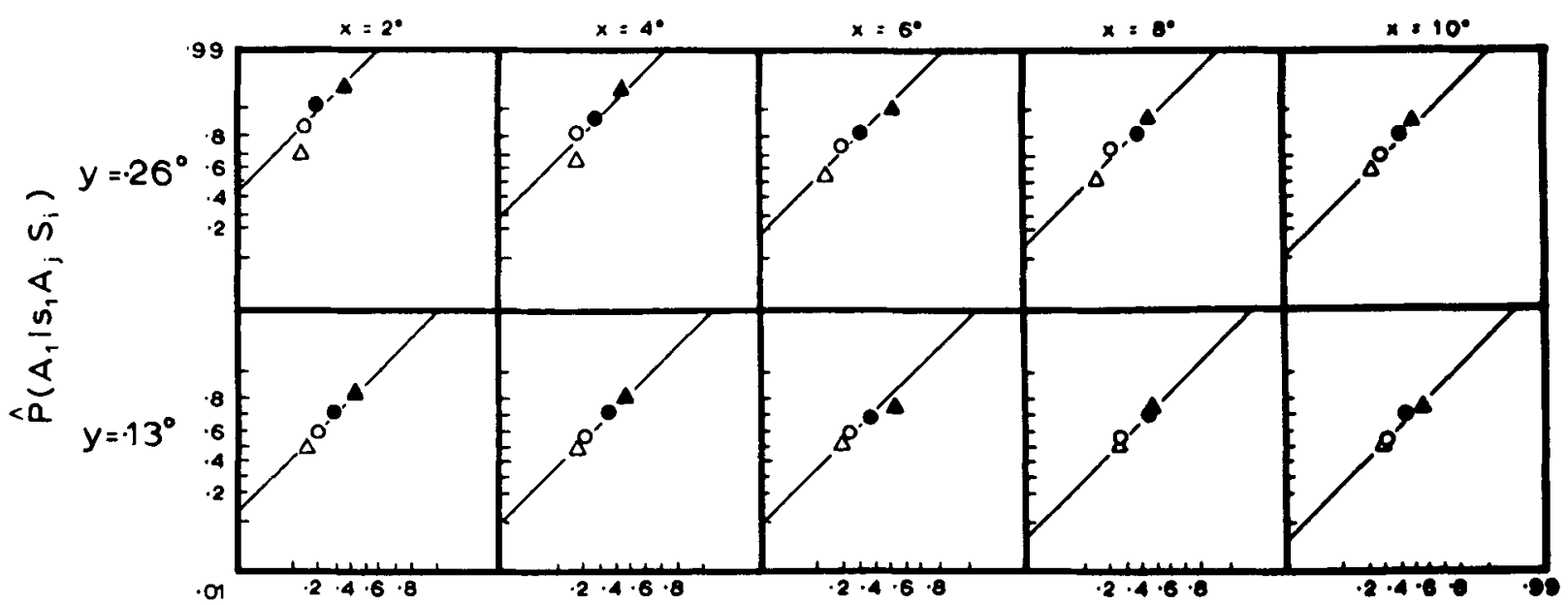

Trial $n-1$

\begin{tabular}{|c|}
\hline $\begin{array}{ll}\triangle A_{1} S_{\circ} & \circ A_{0} S_{0} \\
-A_{1} S_{1} & \triangle A_{0} S_{1}\end{array}$ \\
\hline
\end{tabular}

Fig. 6. Estimated values of $P\left(A_{1} \mid S_{1} A_{j} S_{i}\right)$ and $P\left(A_{1} \mid S_{0} A_{j} S_{i}\right)$ for each experimental condition in Experiment 1.

removal of such idiosyncratic sources of variability from the estimates of the observer's sensitivity.

\section{Sequential Properties of the Data}

Another feature of the data which is of interest are the sequential fluctuations in hits and false alarms. The sequential effects of particular interest concern the correlation between the response on Trial $\mathbf{n}$ and the stimulus and response events on Trial $\mathrm{n}-1$. For each observer the proportion of $\mathrm{A}_{1}$ responses on Trial $n$ contingent upon the stimulus and the response on Trial $n-1$ as well as upon the stimulus event on Trial $\mathrm{n}$ was determined. These proportions were treated as estimates of the following conditional probabilities:

$$
\begin{aligned}
& P\left(A_{1, n} \mid S_{1, n} A_{j, n-1} S_{i, n-1}\right) \\
& P\left(A_{1, n} \mid S_{0, n} A_{j, n-1} S_{i, n-1}\right)
\end{aligned}
$$

for $i$ and $j$ equal to 1 or 0 . The notation in Expressions 15 and 16 can be simplified by omitting the trial subscripts ( $n$ and $n-1$ ). In the remainder of the paper, the temporal order of events in the sequential statistics should be interpreted as corresponding to that in Expressions 15 and 16. Estimates of $P\left(A_{1} \mid S_{1} A_{j} S_{i}\right)$ and $P\left(A_{1} \mid S_{0} A_{j} S_{j}\right)$, denoted as $\hat{P}\left(A_{1} \mid S_{1} A_{j} S_{i}\right)$ and $\hat{P}\left(A_{1} \mid S_{0} A_{j} S_{i}\right)$, for each experimental condition averaged over the six observers are presented on operating characteristic (OC) graphs plotted on double-probability co-ordinates in Figs. 6 and 7. Each point plotted for a given experimental condition characterizes per-

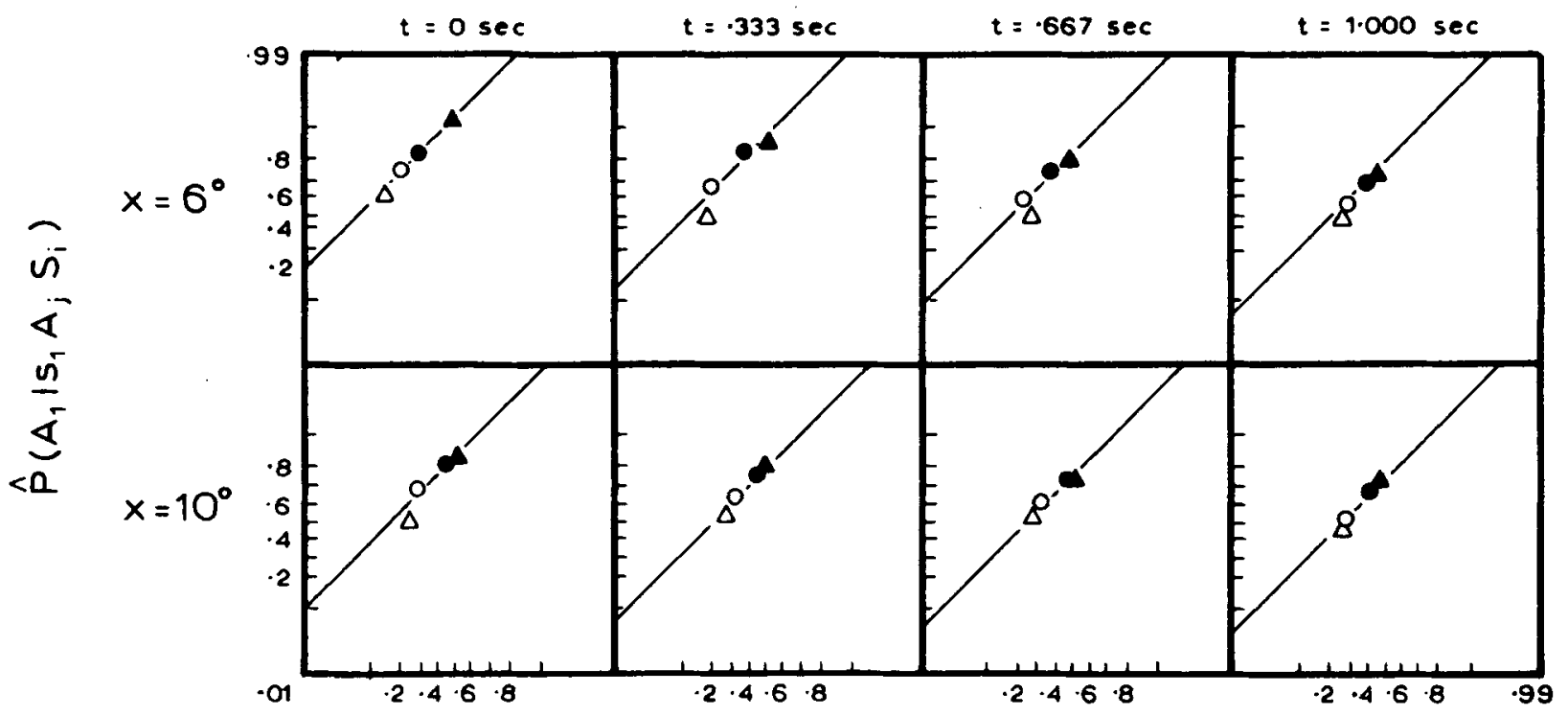

Trial $n-1$
$A_{1} S_{0} \circ A_{0} S_{0}$
- $A_{1} S_{1} \quad \triangle A_{0} S_{1}$
$\hat{P}\left(A_{1} \mid S_{0} A_{j} S_{i}\right)$

Fig. 7. Estimated values of $P\left(A_{1} \mid S_{1} A_{j} S_{i}\right)$ and $P\left(A_{1} \mid S_{0} A_{j} S_{i}\right)$ for each experimental condition in Experiment 2. 


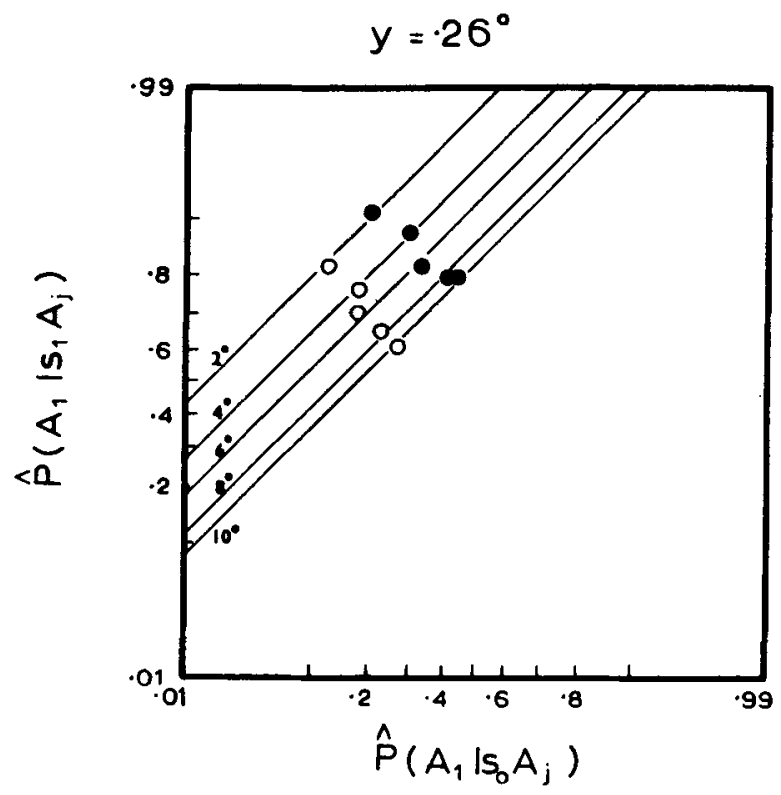

Trial
$-A_{1}$
$\circ A_{0}$

$y=13^{\circ}$

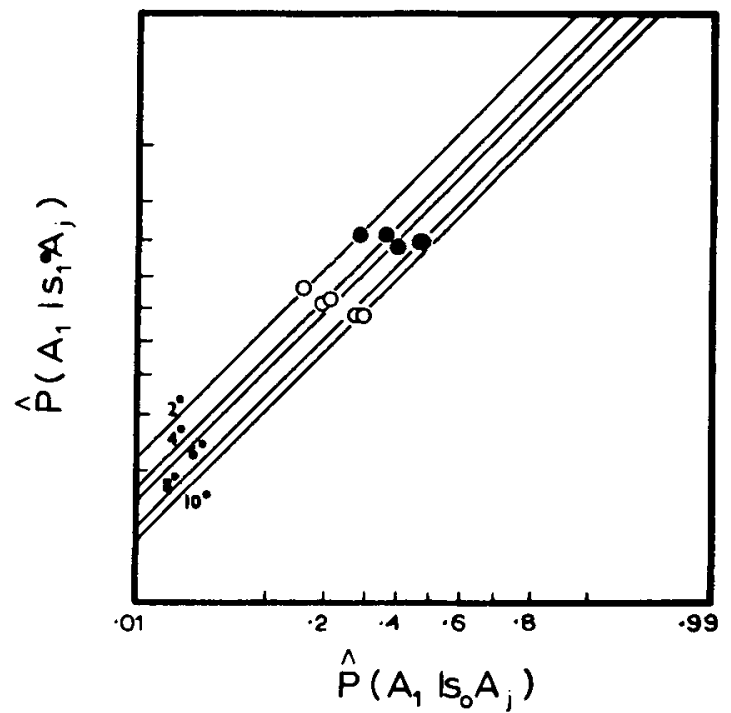

Fig. 8. Estimates values of $P\left(A_{1} \mid S_{1} A_{j}\right)$ and $P\left(A_{1} \mid S_{0} A_{j}\right)$ for each experimental condition in Experiment 1.

formance on trials following each of the four possible combinations of stimulus and response. It is clear that the response on Trial $n$ is correlated with the stimulus and the response events on Trial $n-1$. Note that the order of the four points in the OC space is consistent over all experimental conditions.

These trial-to-trial sequential effects are similar to those described by Kinchla (1966), and Tanner, Haller, and Atkinson (1967) for an auditory recognition problem involving the comparison of stimulus events presented at different points in time. Parducci and Sandusky (1965) reported a study involving the comparison of the horizontal position of two vertically separated visual stimuli. Both the horizontal discrepancy and the vertical separation were constant throughout the experiment. According to Kinchla (1966) a sequential analysis of the Parducci-Sandusky data revealed a similar ordering of points as in the auditory studies involving temporally noncontiguous stimuli. Thus, this relative ordering of the sequential statistics is observed in a number of recognition studies, suggesting that strong sequential effects occur consistently in tasks involving temporally or spatially noncontiguous stimuli. Furthermore, this ordering is consistent with the predictions of a model of signal recognition discussed by

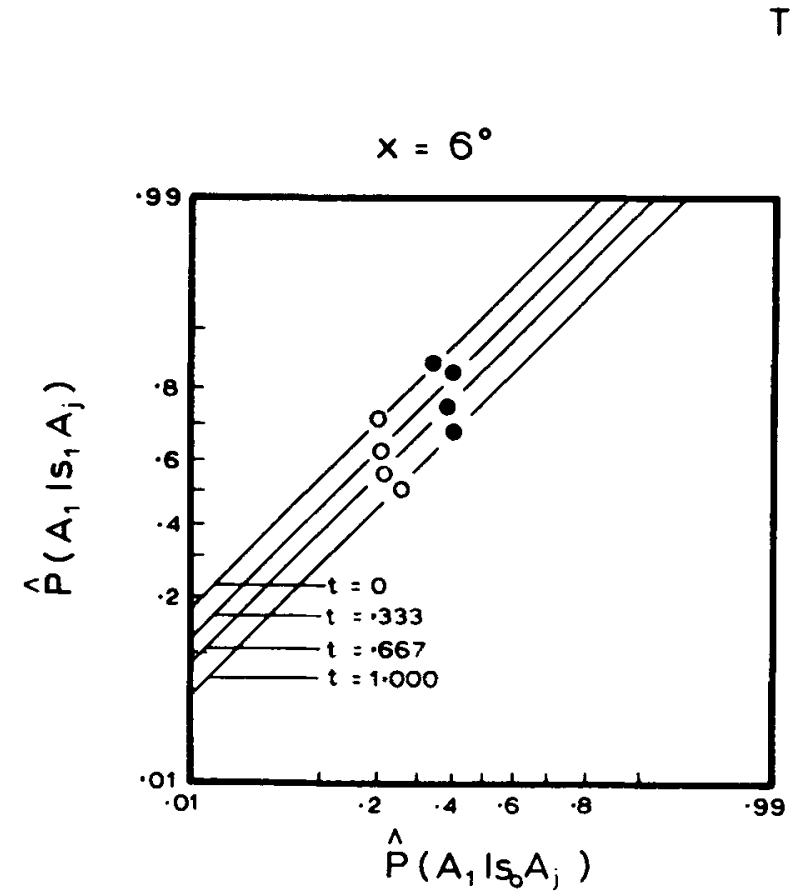

$$
\begin{gathered}
\text { Trial } n-1 \\
-A_{1} \\
\circ A_{0}
\end{gathered}
$$$$
x=10^{\circ}
$$

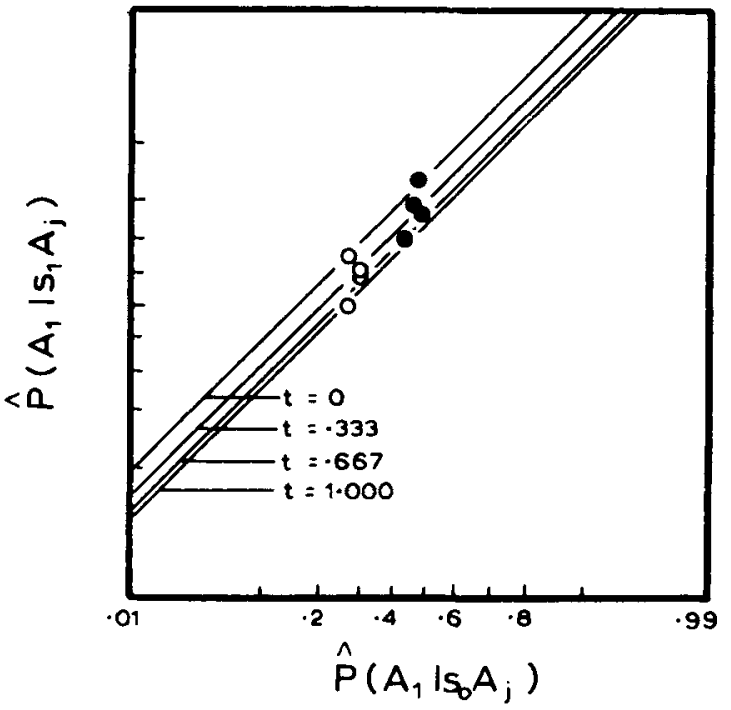

Fig. 9. Estimates values of $P\left(A_{1} \mid S_{1} A_{j}\right)$ and $P\left(A_{1} \mid S_{0} A_{j}\right)$ for each experimental condition in Experiment 2. 
Tanner. Haller, and Atkinson ( 1967 ).

Figures 6 and 7 suggest that, in general, the response on Trial n is more highly correlated with the response on Trial $n-1$ than the stimulus on Trial $n-1$. Estimates of $P\left(A_{1, n} \mid S_{1, n} A_{j, n-1}\right)$ and $\mathbf{P}\left(A_{1, n} \mid S_{0, n} A_{j, n-1}\right)$, denoted as $\hat{P}\left(A_{1} \mid S_{1} A_{j}\right)$ and $\hat{P}\left(\hat{A}_{1} \mid S_{0} A_{j}\right)$, for each experimental condition averaged over the six observers are plotted on double probability co-ordinates in Figs. 8 and 9. Each point plotted for a given experimental condition characterizes performance on trials following each of the two possible responses on Trial $n-1$. The order and the spacing of the two points in the $O C$ space is consistent over all experimental conditions. For each experimental condition a straight line with unit slope provides a very good fit to the two points. Thus, the sequential effects can be interpreted as shifts in decision criterion along the same $O C$ curve. The straight line, unit slope, operating characteristic suggests that the two distributions of $U_{t x}$ (see Fig. 2) have equal variance. This is consistent with one of the assumptions of the model.

For each experimental condition the $O C$ curve for the response contingent sequential effects is replotted in Figs. 6 and 7, and provides an adequate fit to the four points. The most deviant points are generally the most unreliable estimates in that they are based on few observations. It appears then that the observer's decision criterion on Trial $n$ is contingent on both the stimulus and response events on Trial $n-1$.

The model developed in this paper does not specify that the observer shifts his criterion from trial to trial. However, it is clear that since the model accounts for virtually all of the variance in the estimated $\delta_{t x}$ values, the variance due to fluctuations in criterion does not significantly influence $\delta_{t x}$. This point can be made more explicitly in terms of the model. Suppose the criterion was not constant but actually had a Gaussian distribution with mean zero and variance $\phi_{c}$. Then,

$$
\delta_{t x}=\frac{y}{\left(\phi_{t} t+\phi_{x} x+K+\phi_{c}\right)^{1 / 2}}
$$

The results indicate that $\phi_{c}$ is so small relative to the other components of the variance in the denominator of the preceding equation that a good fit between model and data may be achieved with the assumption that $\phi_{c}$ equals 0 (as in Equation 14). It would be of theoretical interest to elaborate the present model to include a dynamic decision process which specifies the manner in which an observer shifts his criterion from trial to trial.

\section{CONCLUSION}

It is clear that the results of the two experiments are generally consistent with the predictions of the model. An observer's ability to compare the vertical position of two points of light presented successively in the dark is reduced by both a temporal delay between their presentation and a lateral separation in their position. The loss of vertical position information during the temporal delay is similar to the loss over a lateral translation in that both can be represented by a random walk process. When the transformation is made simultaneously over both a temporal and a lateral separation, the random walk processes appear to proceed simultaneously but independently, and the cumulative loss of information is simply the sum of both walks. Note that two estimates, $\hat{\phi}_{t}$ and $\hat{\phi}_{x}$, account on the average for $96 \%$ of the variance in $\hat{\delta}_{\mathrm{t} x}$.

It should be emphasized that the discrimination problem considered is somewhat unique in that the loss of vertical position information represented by the theoretical random walk processes may to a large extent reflect overt, involuntary eye movements. The drift components of involuntary eye movements in the dark suggest a random walk process, and probably are the result of instability in the oculomotor system (Cornsweet, 1956). Thus, it is conceivable that the loss of vertical position information during the interstimulus interval, $\phi_{t}$, is due, at least in part, to the instability of the oculomotor system. Similarly, the loss of vertical position information during the lateral translation, $\hat{\phi}_{x}$, might also be attributable, in part, to the instability of the oculomotor system. Unfortunately, there are no reports of direct measurements of eye movements in an experimental situation similar to that used in the present experiments.

It would be of value to apply the model to data from a discrimination problem which is not affected by involuntary eye movements. For example, a task involving the comparison of the brightness of two objects would be appropriate. Specifically, the observer is required to discriminate a difference in brightness between two laterally separated points of light presented successively in the dark. On some trials the two lights would be equated in intensity, on others, they would differ by an amount $y$. In this way it would be possible to test the model in a situation where the variance introduced by a temporal delay and a lateral separation could not be attributed to involuntary eye movements.

Note that this approach would allow one to determine the relation between "brightness memory" and stimulus intensity. According to Weber's Law, an observer's ability to discriminate a change in brightness is related in a systematic manner to the intensity of the stimulus being incremented. Specifically,

$$
\frac{\Delta I}{I}=K
$$

where $I$ is the intensity of the original stimulus, $\Delta I$ is the increment in intensity necessary for the observer to detect a change in brightness, and $K$ is a constant. It has been shown that Weber's Law holds for a fairly wide range of intensities (Dember, 1960). Thus, on the basis of Weber's Law we would expect variations in the intensity of the initial stimulus $\left(\mathrm{y}_{0}\right)$ to influence $\delta_{\mathrm{tx}}$. This suggests that the rate at which brightness information is lost over time $\left(\phi_{t}\right)$ and over space $\left(\phi_{\mathrm{x}}\right)$ could be dependent on the value of $y_{0}$. That is, an observer's ability to "remember" the brightness of an object may be related to the intensity of the object.

An interesting application of the model would be in animal psychophysics. There has been a great deal of interest in an animal's ability to discriminate stimuli which are separated in time. For example, a number of investigators have studied the acquisition of a "delayed matching-to-sample" problem by the pigeon (Berryman, Cumming, \& Nevin, 1963; Blough, 1959; Cumming \& Berryman, 1965). In general, the animal in this problem situation is required to select that one of two "choice stimuli" which is the same as the "sample stimulus." A temporal delay can be introduced between the of fset of the sample stimulus and the presentation of the choice stimuli. It would be interesting to determine whether the predictions of the model are consistent with the poorer matching performance observed in these studies as the temporal delay is increased. Unfortunately, the data reported in these studies are in a form not amenable to a decision theory analysis.

\section{REFERENCES}

ALLAN, L. G. Visual position discrimination: A model relating temporal and spatial factors. Unpublished doctoral dissertation, McMaster University, Hamilton, 1968.

ANDERSON, T. W., \& GOODMAN, L. A. Statistical inference about Markov Chains. Ann. Math. Statist., 1957, 28, 89-110.

ATKINSON, R. C., CARTERETTE, E. C., \& KINCHLA, R. A. Sequential phenomena in psychophysical judgments: A theoretical analysis. IRET Trans. on information theory, 1962, IT-8, 155-162. Reprinted in E. D. Neimark and W. E. Estes (Eds.), Stimulus sampling theory. San Francisco: Holden-Day, 1967. Pp. 601-615.

ATKINSON, R. C., \& KINCHLA, R. A. A learning model for forced-choice detection experiments. Brit. J. math. statist. Psychol, 1965, 18, 183-206.

BERRYMAN, R., CUMMING, W. W., \& NEVIN, J. A. Acquisition of delayed matching in the pigeon. J. exp. Anal Behav., 1963,6,101-107.

BLOUGH, D. S. Delayed matching in the pigeon.J. exp. Anal. Behav., 1959, $2,151.160$.

CORNSWEET, T. N. Determination of the stimuli for involuntary drifts and saccadic eye movements. J. Opt. Soc. Amer, 1956, 46, 987-993.

CUMMING, W. W., \& BERRYMAN, R. The complex discriminated operant: Studies of matching-to-sample and related problems. In D. I. Mostofsky (Ed.), Stimulus generalization. Stanford: Stanford University Press, 1965. Pp. 284-330. 
DEMBER, W. N. Psychology of perception. New York: Holt, Rinehart, \& Winston, Inc., 1960.

GOUREVITCH, V., \& GALANTER, E. A significance test for one parameter isosensitivity functions. Psychometrika, 1967, 32, 25-33.

GREEN, D. M., \& SWETS, J. A. Signal detection theory and psychophysics. New York: Wiley, 1966.

KELLER, W., \& KINCHLA, R. A. Visual movement discrimination. Percept. \& Psychophys, 1968, 3(B), 233-236.

KINCHLA, R. A. A learning factor in visual discrimination. In R. C. Atkinson (Ed.), Studies in mathematical psychology. Stanford: Stanford University Press, 1964. Pp. 233-249.

KINCHLA, R. A. A comparison of sequential effects in detection and recognition. Experimental Psychology Series, New York University, Technical Report No. 1, 1966.

KINCHLA, R. A., \& ALLAN, L. A model for visual movement perception. Psychology Series, McMaster University, Technical Report No. 16, 1968.

KINCHLA, R. A., \& SMYZER, F. A diffusion model of perceptual memory. Percept. \& Psychophys, 1967, 2, 219-229.

PARDUCCI, A., \& SANDUSKY, A. Distribution and sequential effects in judgment. J. exp. Psychol., 1965, 69, 450-459.

RONKEN, D. A. The constant error in amplitude discrimination and the inter-stimulus interval; A re-examination. Psychology Department, University of Washington, Technical Report, 1967.
TANNER, T. A., Jr., HALLER, R. W., \& ATKINSON, R. C. Signal recognition as influenced by presentation schedule. Percept. \& Psychophys., 1967, 2, 349-358.

TANNER, W. P., Jr. Physiological implications of psychophysical data. Annu. N.Y. Acad. Sci., 1961, 89, 752-765. Reprinted in J. A. Swets (Ed.), Signal detection and recognition by human observers: Contemporary readings. New York: Wiley, 1964. Pp. 351-365.

\section{NOTES}

1. This paper is based on a dissertation submitted to the Faculty of Graduate Studies, McMaster University, in partial fulfillment of requirements for the degree of Doctor of Philosophy. The author would like to express her sincere appreciation to Dr. Ronald Kinchla for the stimulation, encouragement, and guidance he provided during all phases of this investigation. Support for this research was provided by the National Research Council of Canada, Grant No. APA-190.

2. Address: Department of Psychology, McMaster University, Hamilton, Canada.

(Accepted for publication July 3, 1968.) 